\title{
SUR LE NOIRGISSEMENT DES VIEILLES CIRES
}

\author{
R. CHAUVIN \\ Station de Recherches sur l'Abeille et les Insectes sociaux, \\ Bures sur Yvette (Seine-et-Oise).
}

\section{SOMMAIRE}

Il existe dans les vieux rayons un ensemble de corps déposés sur la cire, ou à l'état de mélange, qui augmentent beaucoup leur épaisseur et leur densité. Parmi eux on peut dénombrer de la propolis en petite quantité; une substance hydro et alcalisoluble, en quantité plus forte. Cette substance comprend un matériel hydrocarboné, qui réduit le Fehling; un polypeptide phosphoré, et sans doute un corps voisin des mélanines. On ne peut rien dire jusqu'à présent de l'origine de ces substances

Tous les praticiens de 1'Abeille savent que la couleur des cires dans la ruche passe rapidement du jaune brun très clair au brun foncé. Mais il ne semble pas qu'on se soit beaucoup posé de questions sur les causes de ce noircissement, tout au moins à ma connaissance. Au début, la substance qui recouvre les rayons est facilement soluble dans l'acétone qu'elle colore en jaune ; elle se dissout en rose dans le sulfure de carbone et donne avec le trichlorure d'antimoine la réaction caractéristique des caroténoïdes; il s'agit sans doute d'un corps qui provient des poussières de pollen innombrables qui se déposent partout dans la ruche. D'ailleurs, lorsqu'on fait passer les butineuses de retour des champs sur un papier blanc, il est facile de constater que la couleur du papier vire rapidement au jaune rougeâtre.

Mais plus tard un pigment noir se développe qui n'a rien de commun avec les caroténoïdes. Il ne s'agit d'ailleurs pas d'un seul corps mais d'un mélange complexe, comme nous allons le voir. On a admis tacitement qu'il devait provenir de débris variés que les Abeilles incorporent à la cire au cours de plusieurs années; ou encore des excréments larvaires qui sont, comme on le sait, rejetés au-dessous du cocon juste avant la nymphose. Quant à la première hypothèse, il existe sans doute de nombreux débris que la microscope peut mettre en évidence dans la cire (voir travail de RIERA dans le même fascicule) mais un examen mêtme superficiel montre qu'il s'y trouve en outre des substances non figurées et entraînables par divers solvants. En ce qui concerne la seconde hypothèse, il est bien exact que des excréments en quantités non négligeables sont déposées entre les cinq à six cocons empilés dans une cellule âgée d’un rayon ; mais ils se confinent au fond de la cellule; et si l'on regarde la partie inférieure d'un vieux rayon, là où les phénomènes sont particulièrement nets, on se rend compte 
que le bord de la cellule, où n'atteignent pas les excréments, est lui aussi très épais et largement encrassé. De toute façon, des essais d'extraction, même rudimentaires, montrent que le problème est beaucoup plus complexe.

\section{ESSAIS D'EXTRACTION}

Des morceaux de rayons très durs, très épais et noirs, sans miel ni couvain, ont été sélectionnés, écrasés sommairement et épuisés à chaud par un solvant des corps gras (benzène, acétone ou toluène) qui doit enlever les cires ; l'alcool bouillant, qui doit enlever la propolis insoluble dans le benzène ou le toluène ; l'eau chaude ; enfin l'extraction se termine par une macération dans l'eau ammoniacale à $5 \mathrm{p}$. Ioo. Les résultats sont différents, pour le même échantillon de vieux rayons, suivant la méthode employée :

\begin{tabular}{|c|c|c|c|c|c|}
\hline $1^{\text {er }}$ solvant & Cires & Propolis & Hyclrosol. & Alcalisol. & Résidu \\
\hline & $\%$ & $\%$ & $\%$ & $\because$ & $\%$ \\
\hline Benzìne ............ & 18 & '́t & 8 & 6 & 12 \\
\hline Acútone............. & 65 & i) & $s$ & 6 & 14 \\
\hline Toluène ............. & 50 & (i) & 6 & 5 & 16 \\
\hline Benzène (autre échantillon) & 195 & 4,7 & '́t & 3 & 20 \\
\hline
\end{tabular}

On notera des pertes importantes, variables suivant les échantillons et qui tiennent vraisemblablement au fait que les vieux rayons contiennent une quantité importante d'eau difficile à éliminer complètement; j'ai préféré la négliger.

On remarquera aussi la présence d'un extractif hydro-et alcalisoluble important. Les résidus sont essentiellement formés des cocons, qui retiennent encore entre leurs fonds imbriqués, des quantités importantes d'excréments larvaires qu'on ne pourrait extraire complètement que par broyage.

\section{DISCUSSION}

Les cires extraites ont une couleur assez foncée et peuvent évidemment entrainer d'autres matières liposolubles, dont l'étude serait plus compliquée ; je les ai laissées de côté. L'alcool qui a servi à extraire la propolis est à $95^{\circ}$ et entraîne des matériaux hydrosolubles comme on peut s'en assurer en évaporant à sec après l'opération. et en reprenant par l'alcool absolu à chaud : alors la plus grande partie de la propolis passe en solution, mais il reste un résidu brun qui est seulement hydrosoluble. Une partie des dérivés hydrosolubles précipite d'ailleurs par l'alcool. Nous n'avons pas procédé à une étude systématique de la propolis des rayons ; on sait depuis longtemps que les Abeilles ont coutume d'en enduire les cellules et que d'autre part une certaine quantité de cire sa trouve mélangée à la propolis brute qui enduitles cadres. Mais on admettait que la couleur foncée des rayons pouvait être due à cette propolis ; il est facile pourtant de constater que lorsqu'on épuise un rayon par l'alcool bouillant sa couleur foncée reste presque inaltérée et qu'il est encore possible d'en retirer un abondant matériel brun noir hydrosoluble. D'autre part, la propolis de rayons diffère de la propolis de cadres ; d'abord après évaporation de l'alcool qui a servi à l'extraire, elle est privée de l'odeur agréable qui caractérise habituellement cette substance ; corrélati- 
vement, une analyse pratiquée par BARBIER nous a montré la quasi-absence de fractions volatiles distillant vers $80^{\circ}-90^{\circ}$, alors qu'elles sont présentes dans la propolis obtentue par raclage des cadres; d'autre part, et surtout, LAVIE a prouvé que si le pouvoir antibiotique de la propolis de rayon est aussi net que celui de la propolis de cadres, par contre la propolis de rayon n'est pas antifongique (et 1'on sait d'ailleurs que les cadres d'Abeilles moississent assez facilement hors de la ruche) alors que la propolis des cadres l'est fortement (pour le détail des tests employés, voir thèse de LAVIE, I959).

\section{L'extractif hydrosoluble.}

L'extractif hydro- ou alcalisoluble se présente sous la forme d'une liqueur rouge brun très foncée qui concentrée à sec fournit une pâte brun noir très hygroscopique, d'un goût amer et qui, lorsqu'elle est légèrement réhydratée, a la propriété de s'étirer en longs fils; cette particularité se rencontre aussi parmi les pectines; c'est pourquoi mon collègue UL,RICH de la Sorbonne, qui en est spécialiste, a bien voulu les rechercher, et je l'en remercie bien vivement. Malheureusement, la recherche des pectines dans la pâte brun-noir n'a pas donné de résultats positifs. J'ai tenté alors quelques réactions élémentaires :

La liqueur précipite par l'acétate basique et l'acétate neutre de plomb (précipitation incomplète), par le sulfate de cuivre et l'eau de chaux. Elle ne précipite pas par $\mathrm{Na} \mathrm{Cl}$ à saturation ni par $\mathrm{SO}_{4}\left(\mathrm{NH}_{4}\right)_{2}$. La substance réduit fortement le Fehling: sur $500 \mathrm{mg}$ de dérivé on trouve par la méthode de Bertrand $30 \mathrm{mg}$ de sucre calculé en glucose. Mais on n'en trouve pas davantage après une hydrolyse de 3 heures à chaud, en présence de 3 p. roo d'acide sulfurique. On ne peut donc dire qu'il s'agisse d'un hétéroside. Toutes les autres réactions que j'ai tentées, notamment celles des phénols, ne m’ont donné atıcun résultat.

\section{Analyse élémentaire.}

Elle a été pratiquée par mes collègues GuläGen du Laboratoire de Biochimie de 1'I. N. R. A. à Jouy en Josas, et Pozonovski du Laboratoire de Biochimie de la Faculté de Médecine, que je remercie vivement.

On obtient en $\mathrm{g}$ par $\mathrm{kg}$ de matière sèche (GUEGUEN).

\begin{tabular}{|c|c|}
\hline Cendres totales & $(13 \%)$ \\
\hline Phosphore . . . . . . . . . . . . & $(1,5 \%)$ \\
\hline Calcium $\ldots \ldots \ldots \ldots \ldots \ldots \ldots \ldots$ & $(1,3 \%)$ \\
\hline Potassium ................... & $(2,1 \%)$ \\
\hline Sodium $\ldots \ldots \ldots \ldots \ldots \ldots \ldots \ldots$ & $(0,2 \%)$ \\
\hline Carbone..$\ldots \ldots \ldots \ldots \ldots \ldots$ & $34 \%$ \\
\hline Hydrogène $\ldots . \ldots \ldots \ldots \ldots \ldots$ & 5,75 \\
\hline Azote $\ldots \ldots \ldots \ldots \ldots \ldots \ldots \ldots \ldots$ & 2,4 \\
\hline
\end{tabular}

Ia chromatographie exécutée par PoLonovski (butanol - acide acétique-eau : $5 / 3 / \mathrm{I}$ ) montre une abondance de taches colorables à la ninhydrine ce qui correspond à des groupes $-\mathrm{NH}_{2}$ libres, qui peuvent être ceux d'acides aminés ou de peptides; elles se colorent aussi au réactif d'Isherwood, qui signe la présence de dérivés phosphoriques. Mais aucune de ces taches ne se colore par le réactif de Draggendorf ; il n'existe donc pas d'amines quaternaires. Lorsqu'on précipite la liqueur brute par 1'alcool, la 
fraction non précipitable renferme surtout un composé qui donne une grande tache où se trouvent à la fois des groupes $-\mathrm{NH}_{2}$ et $-\mathrm{PO}_{3} \mathrm{H}_{2}$ (phosphopeptide); par électrophorèse sur papier, ce phosphopeptide migre vers la cathode; il est donc plutôt basique.

On se trouve donc en présence, comme il fallait s'y attendre d'un mélange complexe où il semble que l'on puisse distinguer deux groupes de corps principaux $a$ ) des hydrates de carbone, quelle qu'en puisse être la nature, mucilages, gommes, etc. $b$ ) des corps azotés et phosphorés dont un phosphopeptide.

\section{Les dérivés noirs.}

La genèse de l'encrassement des rayons par ces dérivés reste très obscure ; j'avais songé d'abord qu'ils pouvaient venir d'une sécrétion tégumentaire de l'Abeille qui passe des millions de fois sur les rayons lorsqu'on les maintient quelques années dans les ruches. On devrait alors y trouver des traces des mélanines tégumentaires si abondantes chez les ouvrières; en effet comme l'a montré WIGGLESWORTH les abrasions cuticulaires sont très fréquentes chez les insectes du sol par exemple, qui se frottent d'habitude contre des corps durs; il n'est pas impensable qu'elle puisse se produire aussi chez 1'Abeille bien que d'une manière beaucoup moins marquée, car la cire est fort molle. En tout cas des mesures de l'épaisseur de la couche chitineuse chez les jeunes et les vieilles ouvrières ne nous ont pas montré de différences. Mais on peut s'y prendre d'une autre façon ; l'extraction des mélanines du tégument des Abeilles est possible en broyant des ouvrières entières au mixer et en épuisant la pâte ainsi obtenue par l'ac. chlorhydrique pur; on lave avec soin, et on met en contact huit jours avec l'ammoniaque pure; on peut s'attendre à ce que l'acide ait attaqué la chitine, et libère en partie au moins les mélanines qui s'y trouvent incorporées, de manière à ce que l'ammoniaque puisse les dissoudre. On obtient en effet une liqueur brune qui, neutralisée, laisse déposer des flocons noirs. D'autre part, on peut rechercher s'il ne se trouverait point dans les extraits aqueux de rayons vieillis un dérivé analogue. Si on agite vigoureusement avec du noir animal l'extrait aqueux total, lave à l'eau distillée, puis élue avec l'ammoniaque à $5 \mathrm{p}$. Ioo, on obtient une solution noire qui précipite en gros flocons après neutralisation. M. POLONOvSKI a bien voulu en pratiquer l'analyse élémentaire.

\begin{tabular}{|c|c|c|}
\hline & Mélanine des abeilles & Corps noir des cires \\
\hline $\mathrm{C} \%$ & $37,6+$ & 39,18 \\
\hline $\mathrm{H}^{\prime} \ldots \ldots \ldots \ldots \ldots \ldots \ldots \ldots \ldots \ldots \ldots \ldots \ldots$ & 6,71 & 5,83 \\
\hline 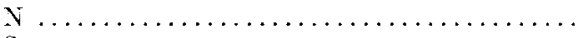 & 7,70 & 8,28 \\
\hline 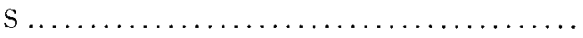 & 7,60 & 5,50 \\
\hline 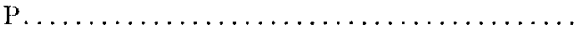 & 0,07 & 0,10 \\
\hline
\end{tabular}

Evidemment, il existe une grande analogie de composition entre les deux substances, surtout en ce qui concerne leur grande richesse en soufre ; ceci ferait songer à une mélanine pour le corps noir des cires, sans que l'on puisse se prononcer encore d'une manière assurée. D'autre part, si l'on pratique l'electrophorèse des deux pigments (Polonovski) on y découvre des différences; celui des rayons présente deux taches : l'une grise qui ne migre pas (on ne la trouve pas dans les mélanines d'Abeilles) l'autre brun rouge, qui migre moins vite que la tache correspondante des Abeilles; 
elle est fluorescente. Le pigment des Abeilles est d'un brun plus foncé, non fluorescent. Il peut s'agir dans le cas du «noir de cire » d'une combinaison de la mélanine avec des sels minéraux.

\section{CONCLUSIONS}

A la phase préliminaire où je me trouve encore, il est évidemment impossible de tirer des conclusions trop affirmatives. Cependant il paraît évident qu'une série de dérivés pratiquement inconnus, quoique très visibles et abondants, se déposent sur les cires. Nous ignorons encore si ces dérivés fortement pigmentés viennent en tout ou en partie des aliments de l'Abeille ou de l'Abeille elle-même. Nous avons vu que la présence d'une mélanine qui proviendrait du tégument des Abeilles est possible, mais non démontrée. D'autre part, les glandes tarsales qui ont été étudiées par ARNHART, servent peut-être à autre chose qu'à 1'adhésion au substrat, comme le voulait cet auteur. Nous avons des raisons de penser que leur sécrétion est liposoluble, et abondante ; elle pourrait donc encrasser les cires sans que l'on puisse l'en séparer.

Pour le savoir, j'ai découpé aussi exactement que possible trois fragments de rayon égaux quant à leurs dimensions $(13,5 \times 7,5 \mathrm{~cm}$, épaisseur $2,5 \mathrm{~cm})$. I'un, $\mathrm{A}$, est jaune pâle et léger; le second, $B$, brun foncé ; C est brun-noir et très lourd.

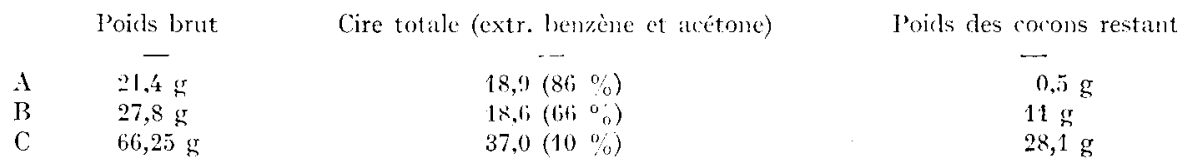

Pour la même surface, par conséquent, les abeilles ajoutent bien de la cire au rayon, bien que le pourcentage de la cire au poids total diminue.

Reçu pour publication on juin 1961.

\section{SUMMARY}

BLACKENING OF OLD WAX

There is in old combs aggregated substances deposited on the wax, increasing its thickness and density. Among these has been found a small amount of propolis, and a larger amount of a hydroand alkali- soluble substance. This substance contains a carbohydrate material reducing Fehling solution, a phosphorus polypeptide, undoubtedly a substance related to melanins. So far nothing can be said as to the origin of these substances.

\section{RÉFÉRENCES BIBLIOGRAPHIQUES}

ARviART L., 1923. Arch. Bienenk., 5, 37-87

Lavie P., rgoo Les substances antibactériennes dans la colonnie d'Abeilles. Thèse Paris, r9o 1), Ann. Abeille, 3 